\title{
Beyond the abstract-concrete dichotomy: Mode of acquisition, concreteness, imageability, familiarity, age of acquisition, context availability, and abstractness norms for a set of 417 Italian words
}

\author{
Pasquale A. Della Rosa \\ San Raffaele Vita-Salute University, Milan, Italy \\ and University of Geneva, Geneva, Switzerland \\ Eleonora Catricalà \\ San Raffaele Vita-Salute University, Milan, Italy \\ and Milano-Bicocca University, Milan, Italy \\ Gabriella Vigliocco \\ University College London, London, England \\ AND \\ STEFAno F. CAPPa \\ San Raffaele Vita-Salute University, Milan, Italy
}

\begin{abstract}
The main objective of this study is to investigate the abstract-concrete dichotomy by introducing a new variable: the mode of acquisition (MoA) of a concept. MoA refers to the way in which concepts are acquired: through experience, through language, or through both. We asked 250 participants to rate 417 words on seven dimensions: age of acquisition, concreteness, familiarity, context availability, imageability, abstractness, and MoA. The data were analyzed by considering MoA ratings and their relationship with the other psycholinguistic variables. Distributions for concreteness, abstractness, and MoA ratings indicate that they are qualitatively different. A partial correlation analysis revealed that MoA is an independent predictor of concreteness or abstractness, and a hierarchical multiple regression analysis confirmed MoA as being a valid predictor of abstractness. Strong correlations with measures for the English translation equivalents in the MRC database confirmed the reliability of our norms. The full database of MoA ratings and other psycholinguistic variables may be downloaded from http:// brm.psychonomic-journals.org/content/supplemental or www.abstract-project.eu.
\end{abstract}

One of the most intriguing questions in cognitive science concerns the way in which concepts are acquired and represented in the mind. It is still a matter of debate whether abstract and concrete concepts are represented in the same way, since the definition of what is concrete and what is abstract is quite vague. The first concept refers to something tangible that we are able to perceive through our senses, whereas the second refers to entities that we cannot perceive directly through our senses.

Two main theories have been developed to account for the difference between abstract and concrete concepts: dual-coding theory (Paivio, Yuille, \& Madigan, 1968) and context availability theory (Schwanenflugel, Harnishfeger, \& Stowe, 1988). The first hypothesis claims that there exists a dual-coding system responsible for the storage of the semantic representations related to concepts. One is grounded in information derived from our perceptual experience, whereas the other is based on verbal information derived from language. According to this position, the differences between abstract and concrete concepts can be ascribed to the different availability of the two systems for concrete concepts (perceptual and verbal) with respect to abstract ones (only verbal). The second theory posits the existence of a single coding system for the two types of concepts and states that differences are due to the quantity of contextual information and the availability of the context for both types of concepts. Both theories predict that there are differences between abstract and concrete concepts that may be mirrored by different cognitive processes.

Different psycholinguistic variables have been defined to quantify and measure the differences between these

P. A. Della Rosa, dellarosa.pasquale@hsr.it 
two classes of concepts. The most important ones are concreteness (CNC), imageability (IMG), context availability (CA), familiarity (FAM), and age of acquisition (AoA). All these variables have been investigated through rating procedures, in which participants are asked to evaluate words with respect to each measure and to assign a score to each word, which represents a measure of a particular concept with respect to the specific variable (Altarriba, Bauer, \& Benvenuto, 1999; Setti \& Caramelli, 2005; Wauters, Tellings, van Bon, \& van Haaften, 2003; WiemerHastings, Krug, \& Xu, 2001).

The results of these studies have shown clear differences between abstract and concrete concepts when the distribution of the words belonging to both classes on each of the scales related to the different variables were considered. Concrete words were usually assigned higher scores with respect to $\mathrm{CNC}, \mathrm{IMG}$, and $\mathrm{CA}$ than were abstract concepts (Altarriba et al., 1999). Furthermore, there were significant correlations between IMG and CNC (Paivio, 1971) and between CA and CNC (Schwanenflugel et al., 1988). Abstract concepts also appeared to depend heavily on CA (Schwanenflugel, Akin, \& Luh, 1992).

The common sense notion underlying all these variables is that an entity is abstract when we cannot perceive it and that concepts are more and more abstract to the degree they get less perceivable (concrete), less imageable, or more difficult to contextualize. However, the fact that concrete knowledge is grounded in the perceptual (sensory-motor) experience of the world does not necessarily imply its contrary - that is, that all concepts that are not grounded in our senses are abstract.

A promising way to progress beyond the dichotomous view is to take into account our conception of how we experience abstract concepts. The general aim of this study was to explore the abstract domain of conceptual knowledge by introducing a new variable, mode of acquisition (MoA). The concept of MoA was first introduced by Wauters et al. (2003), and it is grounded in the assumption that the meaning of a word can be acquired perceptually, linguistically, or by a combination of both. The meaning of a word may be depicted as the interplay between different associations that are established with that word. The nature of these associations may take a linguistic form (e.g., "love is a feeling that leads a man and a woman to marriage and having a baby") or lie in a perceptual input that we may experience through our senses or an internal state (e.g., a mother hugging or kissing her child, who will feel happy).

A concept is built on both perceptual and linguistic information. The combination of both types of information subtends the knowledge structure underlying the meaning of words and constantly "adds pieces" to the core of a concept. In this sense, the word love will mean a mother hugging or kissing her child, which will become a man kissing a woman, which will become a couple getting married, with a baby being the "fruit of their love."

MoA is a property measured just like AoA, CNC, IMG, FAM, and CA. MoA is surely related to all these characteristics; however, it holds an advantage over all of them in the fact that it imprints the meaning of a concept (through experience or language) but is sensitive to the contextual frame in which the concept is acquired as well. For example, children born in Australia will acquire the meaning of kangaroo through their senses, whereas children living in Alaska more likely will acquire its meaning through language in a very high proportion. Thus, MoA seems to convey both internal (from the concept) and external (from the context) sources of information to word meanings.

The relationship between MoA and abstractness (ABS)/ $\mathrm{CNC}$ becomes clear if MoA is considered a variable able to weigh the amount of experience and the language shaping the core meaning of a concept. The prevalence of one or the other type of information may give a more concrete or a more abstract label to word content (Gleitman, Cassidy, Nappa, Papafragou, \& Trueswell, 2005) and determine whether the same word is more or less contextually constrained.

In their study, Wauters et al. (2003) asked 25 students to rate MoA for 500 words extracted from school textbooks used in elementary classes in order to outline reading comprehension difficulties in deaf children through elementary school. Their findings showed that MoA ratings gradually change over grades, shifting from words acquired mainly through perception in books for lower grades to words learned mainly through language in Grade 6 texts. A significant correlation $(r=.59, p<.01)$ was also found between MoA and AoA and was accounted for by the fact that younger children are in an earlier stage of language development. However, AoA failed to completely predict MoA and vice versa, since only $35 \%$ of the variance was explained by some relation between the two constructs, which suggests that they are strongly interrelated but do not coincide.

In a recent study, Kousta, Della Rosa, Cappa, and Vigliocco (2007) tried to disentangle the relation between MoA and AoA and to establish the independence and validity of MoA. They asked 146 participants to rate 1,975 words on MoA and used linear regression to analyze lexical decision reaction times (RTs) for 1,881 words collected in the English Lexicon Project (Balota et al., 2007) to examine the predictive power of MoA. The authors found that even though MoA and AoA were significantly correlated $(r=.85)$, MoA predicts lexical decision RTs $\left(R^{2}=\right.$ $.726, \beta=.11)$, even when AoA effects were partialled out, whereas the reverse was not true ( $\beta=.046)$. Furthermore, the correlation between MoA and RTs showed that there was a processing advantage for words acquired through experience with the world over words acquired through experience with language. They concluded that MoA and AoA are independent and that MoA is a valid construct that is able to predict lexical decision RTs. Thus, MoA may be considered as a meaning-related variable, since lexical decision is more sensitive to semantic variables.

In the present study, participants were asked to rate a set of abstract and concrete concept nouns on seven dimensions (CNC, IMG, CA, FAM, AoA, MoA, and ABS). The ABS dimension was added because it was supposed that it would capture variations in the degree of ABS better than would the $\mathrm{CNC}$ dimension.

The present study had five main objectives. The first was to present MoA ratings for 210 concrete nouns and 
207 abstract nouns in Italian that might serve to aid future research on the representation of different types of knowledge in semantic memory. In addition, a correlational analysis was performed to investigate the relationship of MoA with a number of other psycholinguistic variables (CNC, IMG, CA, FAM, AoA, ABS), in order to assess the specificity of MoA. Concerning the semantic characteristics of words for this language, only AoA, FAM, IMG, and $\mathrm{CNC}$ ratings added with various frequency measures; other lexical and sublexical variables are provided for 626 Italian nouns and are available in the LEXVAR database (Barca, Burani, \& Arduino, 2002). The second aim was to assess the form of the distribution of $\mathrm{CNC}, \mathrm{ABS}$, and MoA ratings, in order to evaluate whether ratings for abstract and concrete nouns are evenly distributed across all three scales or whether they group into distinct clusters on one dimension rather than on the others, pointing to the existence of distinct classes of entities. The third aim was to assess the independence of MoA-in particular, with respect to AoA in terms of their relation to $\mathrm{CNC}$ and ABS ratings. The fourth aim was to evaluate the reliability of the present norms by considering the correlation of CNC, IMG, FAM, and AoA norms for the whole set of words (417) in our database with previously published norms for the same set of translation equivalents in English, for which ratings are available in the MRC database (Coltheart, 1981). Finally, a fifth objective was to present a database for all the dimensions analyzed, for future use in research.

\section{Method}

Participants. Ratings were obtained for the seven variables (CNC, IMG, CA, FAM, AoA, MoA, and ABS) from a sample of 250 university students majoring in psychology, biology, physiotherapy, medicine, and nursing. All of the participants were native speakers of Italian.

Materials. We selected a set of 417 Italian words (210 concrete and 207 abstract words) corresponding to translation equivalents of a list of 1,975 English words extracted from the MRC database (Coltheart, 1981) and used in a previous study in English (Kousta et al., 2007). Subsets of concrete and abstract words were selected according to three reference ranges of $\mathrm{CNC}$ (low, medium, and high $\mathrm{CNC}$ ). Within the subset of abstract words, 70 words that refer to emotions were included, since previous studies had claimed that this class of words is different with respect to highly concrete and highly abstract words in terms of distribution, according to the variables investigated here (Altarriba et al., 1999).

In order to identify abstract and concrete words with low, medium, and high $\mathrm{CNC}$ values, the observed 5th, 25th, 50th (median), 75th, and 95th percentiles were calculated for words below and above the median of the distribution of the whole MRC data set on the basis of the empirical distribution of the data on the CNC scale in the MRC. The percentiles used to classify the different levels of $\mathrm{CNC}$ are re- ported in Table 1. Specific ranges for abstract and concrete words were established, since the distribution of the words on each end of the scale cluster differently within the interpercentile ranges.

Procedure. The set of 417 words were inserted into different rating lists. Seven lists of 60 words ( 30 abstract words +30 concrete words) were created, in order to rate the whole set of 417 words. The lists were rotated among the seven dimensions, so that each word could be rated on all the dimensions. Each participant was presented with seven lists of words corresponding to the seven different dimensions and rated the whole set of 417 words only once through the seven dimensions. This procedure allowed us to collect data for all the words on all the scales. Each word was rated on each scale by an average of 35 people. The order of the words within every list was randomized (three different list versions for each variable were created), and the order of the lists for each variable was randomized across participants.

All consent information and instructions for the tasks were provided in written Italian. The lists were distributed among seven different groups of participants. The participants were also permitted to stop the rating at any time and to restart at another time, as long as they continued and handed in the list within a week's time.

The scales for CNC, IMG, FAM, CA, ABS, and MoA ranged from 1 to 7, in which 1 indicated highly abstract, difficult to image, unfamiliar, difficult to think of a context, less abstract, and totally acquired through experience and 7 indicated highly concrete, easy to image, familiar, easy to think of a context, more abstract, and totally acquired through language. For AoA, instead, the participants were asked to estimate the age at which they thought that they had learned each word. The Italian version of the instructions used for each variable may be downloaded from www.abstract-project.eu. The instructions for CNC, IMG, FAM, CA, and AoA were based largely on those used by previous investigators (Campos, 1990; Friendly, Franklin, Hoffman, \& Rubin, 1982; Gilhooly \& Logie, 1980; Pavio et al., 1968; Schwanenflugel et al., 1992; Toglia \& Battig, 1978). They were readapted and translated into Italian. The instructions for MOA were specifically created for a previous study performed in an English population (Kousta et al., 2007) and translated into Italian.

Each participant's responses were coded and saved as Microsoft Excel files. We first examined their responses, to ensure that each participant had understood the instructions and completed the ratings for each dimension adequately after having given his or her consent to participate. The data for several participants were discarded as a result of lack of motivation or failure to follow instructions. We used two different criteria in order to exclude participants. The first was to exclude participants who used the same response (e.g., 7) for more than $85 \%$ of the total responses for each list. The second took into account participants' scores that were more than 3 standard deviations away from the groups' average for each item. A mean of 2.38 participants (taking into account the number of participants excluded for the three list versions created for the rating procedure) for each variable were excluded. Next, we extracted the participants' ratings for each of the seven dimensions. For CNC, IMG, FAM, CA, ABS, and MoA, the participants' responses were simply values on a 1-7 scale and required no further processing. For AoA, we converted ratings from numeric values into 7-point scale values. The scale ranged from 1 (age, $0-2$ years) to 7 (age, 13 years and older). Intermediate points on the scale were identified with 2-year age bands.

Table 1

Classification of Low, Medium, and High Concreteness (CNC) for Abstract and Concrete Words on the CNC Scale in MRC

\begin{tabular}{|c|c|c|c|c|c|c|}
\hline \multicolumn{3}{|c|}{ Abstract Words } & \multirow[b]{2}{*}{ CNC Median } & \multicolumn{3}{|c|}{ Concrete Words } \\
\hline 5 th-10th & 15 th -45 th & 50 th-75th & & 10th-40th & 45th-75th & 75th-95th \\
\hline $\begin{array}{l}\text { Low } \\
\text { concreteness }\end{array}$ & $\begin{array}{l}\text { Medium } \\
\text { concreteness }\end{array}$ & $\begin{array}{l}\text { High } \\
\text { concreteness }\end{array}$ & & $\begin{array}{l}\text { Low } \\
\text { concreteness }\end{array}$ & $\begin{array}{l}\text { Medium } \\
\text { concreteness }\end{array}$ & $\begin{array}{l}\text { High } \\
\text { concreteness }\end{array}$ \\
\hline
\end{tabular}

Note-Percentile ranges from which abstract and concrete words were sampled are shown. 
The responses for all the participants were coded and merged into a database, and values for each word on each of the seven dimensions were multiplied by 100 to produce a range from 100 to 700 (Coltheart, 1981).

\section{Results and Discussion}

Mean MoA ratings and their respective standard deviations are presented in the full database by alphabetical order in Italian, along with their English translation. The full database of MoA ratings and other psycholinguistic variables may be downloaded in the supplement for this article at http://brm.psychonomic-journals.org/ content/supplemental, or from www.abstract-project.eu (where possible, updates to the database will be posted). The mean rating for each word on each of the seven dimensions is reported in the database. Table 2 presents the means, standard deviations, minimums, and maximums for the different variables. Table 3 presents the correlations of the different variables with MoA. Item values of the different measures are presented in the full database.

For the total sample, the mean MoA was 380, with a range from 110 to 663 and a standard deviation of 154 . Overall, correlations of MoA with the other dimensions show a substantial degree of collinearity among these variables (substantially intercorrelated variables). The correlation between MoA and CNC was $-.84(p<.01)$, and that between MoA and IMG was $-.84(p<.01)$, indicating that words with a high $\mathrm{CNC}$ rating or a high IMG rating tend to have low MoA ratings, suggesting that their meanings are acquired mainly through perceptual experience. MoA has negative correlations also with FAM $(r=-.68$, $p<.01)$ and CA, suggesting that words acquired through experience tend to be considered as highly familiar and to be associated with more contextual information.

MoA also appears to be highly related to AoA $(r=.83$, $p<.01)$. This is not unexpected, since younger children

Table 2 Descriptive Statistics on Independent Variables

\begin{tabular}{llcccc}
\hline \multicolumn{1}{c}{ Variable } & Code & Mean & SD & Min & Max \\
\hline Concreteness & CNC & 464 & 196 & 132 & 700 \\
Imageability & IMG & 484 & 183 & 129 & 700 \\
Familiarity & FAM & 531 & 101 & 253 & 700 \\
Age of acquisition & AoA & 326 & 114 & 118 & 606 \\
Context availability & CA & 519 & 127 & 200 & 700 \\
Abstractness & ABS & 312 & 180 & 100 & 635 \\
Mode of acquisition & MoA & 380 & 154 & 110 & 663 \\
\hline
\end{tabular}

Note-Min, minimum value; Max, maximum value.

Table 3

Correlations of Mode of Acquisition With the Variables

\begin{tabular}{lr}
\hline Variable & \multicolumn{1}{c}{$r^{* *}$} \\
\hline CNC & -.85 \\
IMG & -.85 \\
FAM & -.62 \\
AoA & .83 \\
CA & -.86 \\
ABS & .83 \\
\hline
\end{tabular}

Note-CNC, concreteness; IMG, imageability; FAM, familiarity; AoA, age of acquisition; CA, context availability; ABS, abstractness. ${ }^{* *}$ All correlations are significant at the .001 level (two-tailed). will tend to rely more on perceptual inputs than on linguistic information when acquiring the meaning of a concept. In contrast, older children in later stages of language development will exploit the properties of language to build conceptual representations. However, notwithstanding the significant correlation between MoA and AoA in our set of 417 words, AoA ratings are not able to completely predict MoA, since only $35 \%$ of the variance is explained by some relation between the two variables when all the information deriving from the other constructs is controlled for. The remainder of the variance is not explained by AoA, and the percentage related to AoA is the same as the one reported in Wauters et al. (2003), in which MoA ratings and AoA ratings were compared for 444 words.

The empirical distribution of the whole set of 417 words on each of the seven scales was plotted (CNC, ABS, and MoA distributions are shown in Figure 1). Ratings of CNC (top left panel of Figure 1) were bimodally distributed, in agreement with other studies (Nelson \& Schreiber, 1992; Weimer-Hastings et al., 2001). The bimodal distribution is consistent with the view that abstract and concrete entities form two fairly distinct clusters according to particular characteristics (e.g., tangibility or visibility). Within these two clusters, entities vary in $\mathrm{CNC}$, but the distribution skews toward the abstract pole, showing that $\mathrm{CNC}$ fails to account for the variation in the degree of abstractness (see the top left panel of Figure 1).

In contrast, ABS ratings (top right panel of Figure 1) were heavily skewed toward the lower end of the scale (less abstract), where all the concrete words are clustered (see the top right panel of Figure 1). However, ABS seemed to capture differences between abstract entities, at least as far as the degree of perceptual information in which these are grounded.

MoA ratings (bottom left panel of Figure 1) were distributed across the scale, indicating that participants tended to use the entire range of possible combinations of information underlying the acquisition of a concept. These could be totally learned through experience, through language, or through a mixture of both, irrespective of the distinction between abstract and concrete dimensions. However, when the two samples of abstract and concrete words are considered, there is a significant difference between their mean ratings on the MoA dimension $(t=25.13, p<$ $.0001)$, highlighting that the meaning of concrete words is acquired mainly through experience $(M=262, S D=$ $105)$, whereas knowledge related to abstract entities is more grounded in language $(M=500, S D=87$; see the bottom right panel of Figure 1).

Associations between MoA and CNC ratings and between MoA and ABS ratings were quantified using partial correlation coefficients, taking into account possible associations with IMG, FAM, CA, and especially AoA. In this context, a partial correlation between MoA and CNC or between MoA and ABS means that IMG, FAM, $\mathrm{CA}$, and AoA are eliminated from the association. Partial correlation coefficients for $\mathrm{MoA}$ and $\mathrm{CNC}$, as for $\mathrm{MoA}$ and ABS, revealed significant statistical associations between MoA and the CNC $(c c=-.249, p<.0001)$ or 

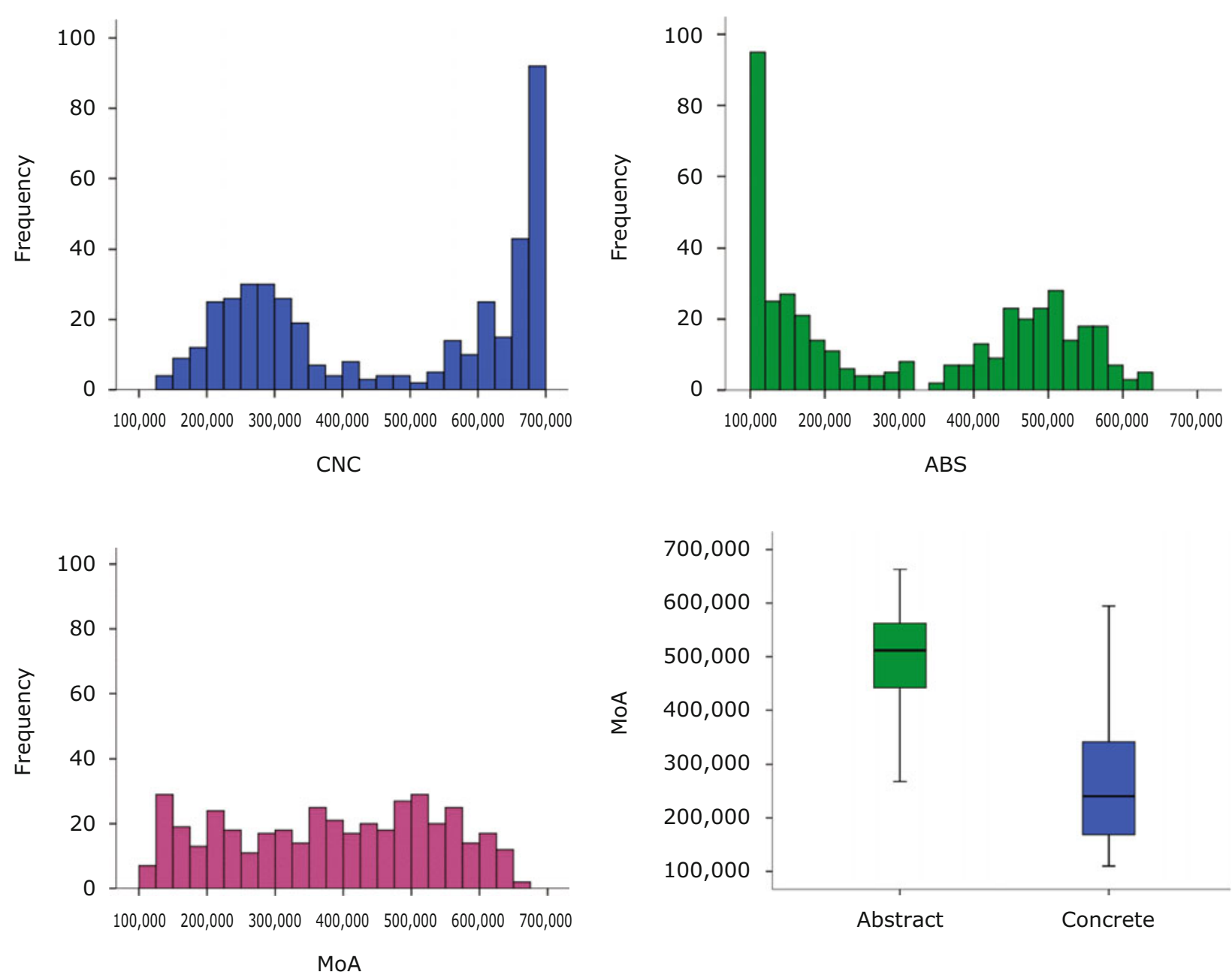

Figure 1. Frequency histograms reflecting the distribution of concreteness (CNC; top left panel), abstractness (ABS; top right panel), and mode of acquisition (MoA; bottom left panel) ratings for the 417 Italian words normed here. A boxplot representing the differences between abstract and concrete words in terms of MoA is shown in the bottom right panel.

the ABS $(c c=.203, p<.0001)$ dimension. However, partial correlation coefficients between AoA and CNC $(c c=.015, p=.758)$, as for AoA and ABS $(c c=-.009$, $p=.853)$, failed to reach significance after adjustment for IMG, FAM, CA, and MoA (see Table 4). In order to establish the independence of MoA with respect to AoA and assess its unique relationship with ABS, we used hierarchical multiple regression in order to determine whether MoA accounts for a significant amount of additional variance of the ABS dimension over AoA and above IMG, CA, and FAM by testing the $R^{2}$ change for statistical significance. At each step of the regression, we highlight the contribution of the variables entered at that step, controlling for the effects of the variables entered at the preceding step. The change in $R^{2}$ at each step determines whether the variables entered account for

Table 4

Partial Correlation Analysis

\begin{tabular}{|c|c|c|c|c|}
\hline & \multicolumn{2}{|c|}{ Concreteness } & \multicolumn{2}{|c|}{ Abstractness } \\
\hline & Unadjusted & $\begin{array}{c}\text { IMG, FAM, CA, } \\
\text { AoA Adjusted }\end{array}$ & Unadjusted & $\begin{array}{l}\text { IMG, FAM, CA, } \\
\text { AoA Adjusted }\end{array}$ \\
\hline \multirow[t]{2}{*}{ Mode of acquisition } & $-.85^{* *}$ & $-.249^{* *}$ & $.83^{* *}$ & $.203^{* *}$ \\
\hline & Unadjusted & $\begin{array}{l}\text { IMG, FAM, CA, } \\
\text { MoA Adjusted }\end{array}$ & Unadjusted & $\begin{array}{l}\text { IMG, FAM, CA, } \\
\text { MoA Adjusted }\end{array}$ \\
\hline Age of acquisition & $-.76^{* *}$ & .015 & $.75^{* *}$ & -.009 \\
\hline
\end{tabular}

Note-Pearson's correlation coefficients and partial correlation coefficients after adjustment are shown. IMG, imageability; FAM, familiarity; CA, context availability. ${ }^{* *}$ All correlations are significant at the .001 level (two-tailed). 
Table 5

Hierarchical Multiple Regression Analysis With Three Steps

\begin{tabular}{lccc}
\hline Step Variables & $R^{2}$ Change & $\beta$ & $p$ \\
\hline 1. IMG, CA, FAM & .914 & & \\
IMG & & -.876 & .000 \\
CA & & -.158 & .017 \\
FAM & & .116 & .002 \\
2. AoA & .001 & .060 & .168 \\
3. MoA & .004 & .159 & .000 \\
\hline
\end{tabular}

Note $-\beta$, unstandardized beta; $p, p$ value for significant $F$ change; IMG, imageability; CA, context availability; FAM, familiarity; AoA, age of acquisition; MOA, mode of acquisition; dependent variable = abstractness.

significant variation in $\mathrm{ABS}$ or, in other words, whether variance in $\mathrm{ABS}$ is significantly cut down when IMG, CA, and FAM are entered at Step 1, then AoA at Step 2, and then MoA at Step 3. Specifically, the change in $R^{2}$ estimates how much predictive power is added to the model by the addition of AoA first and then MoA to the initial set of predictors of ABS (IMG, CA, and FAM). $F$ tests are then computed to assess whether the inclusion of AoA or MoA results in a statistically significant increment in $R^{2}$-namely, a significant $F$ change.

Change in $R^{2}$ for each step of the analysis, as well as unstandardized beta weights and $p$ values for each predictor in the final model, are presented in Table 5. As was expected, IMG, CA, and FAM are all significantly related to ABS, as summarized in Table 5. The contribution of AoA was examined at Step 2, and interestingly, the variance in ABS was not significantly reduced $\left(R^{2}\right.$ change $<$ $.001, F$ change $=1.9, p=.168$ ).

At Step 3, instead, when MoA was added to the model, it accounted for a significant proportion of variance $\left(R^{2}\right.$ change $=.004, F$ change $=17.7, p<.001)$.

Regarding the correlation of the present ratings to the $\mathrm{MRC}$, as was mentioned previously, four variables are in common in the two databases: CNC, IMG, FAM, and AoA. Similar instructions have been used to collect norms. One difference between the two databases resides in the fact that values for English words result from merging different sets of existing norms, whereas norm collection for Italian nouns was specifically addressed in creating this database. The pattern of correlations among the values provided by our sample and the English values is high and significant for all four variables. The list of the correlation coefficients for the measures from the two databases is reported in Table 6.

Table 6

Correlations With Measures for the English Translation Equivalents in the MRC Database

\begin{tabular}{cc}
\multicolumn{2}{c}{ Equivalents in the MRC Database } \\
\hline MRC Variable & $\begin{array}{c}\text { Correlation With Present Study } \\
\left(r^{* *}\right)\end{array}$ \\
\hline CNC & .96 \\
IMG & .90 \\
FAM & .68 \\
AoA & .82 \\
\hline
\end{tabular}

Note-CNC, concreteness; IMG, imageability; FAM, familiarity; AoA, age of acquisition. ${ }^{* *}$ All correlations are significant at the .001 level (two-tailed)

\section{Conclusions}

In the present study, we collected CNC, IMG, FAM, AoA, CA, ABS, and MoA ratings for a total of 417 nouns in Italian and evaluated the relationship of MoA with the other psycholinguistic variables.

Correlation results show that MoA is related to all the variables included in this study, emphasizing, in particular, the association between MoA and AoA ratings. Nevertheless, MoA can be differentiated from AoA, since it is not fully predicted by AoA values, and additional evidence provided by a partial correlation analysis and a subsequent hierarchical regression analysis procedure confirms that they are different. This is in agreement with the results of previous studies (Kousta et al., 2007; Wauters et al., 2003).

An important and unexpected result related to the difference between abstract and concrete knowledge is that both $\mathrm{CNC}$ and $\mathrm{ABS}$ distributions are qualitatively different with respect to the MoA distribution. Concrete and abstract words on the $\mathrm{CNC}$ and ABS dimension are represented by a bimodal distribution, whereas on the MoA dimension, they form a continuum. Furthermore, the difference in terms of MoA ratings between abstract and concrete words suggests that concrete words are acquired mainly through experience, whereas the meaning of abstract words is tightly bound to language.

MoA and AoA have a similar relationship with $\mathrm{CNC}$ and ABS. They are both negatively correlated with $\mathrm{CNC}$, while showing a positive correlation with ABS. This means that concrete words are associated with experience and a lower AoA, whereas abstract words are coupled more with language and are acquired later in time.

It is also noteworthy that the correlation between $\mathrm{MoA}$ and $\mathrm{CNC}$, as well as the one between MoA and ABS, remained significant after adjustment for IMG, FAM, CA, and AoA. In contrast, the relationship between AoA and both $\mathrm{CNC}$ and $\mathrm{ABS}$ disappears after the same adjustments. This finding suggests that only MoA is an independent predictor of $\mathrm{CNC}$ or ABS. Furthermore, when all the variables were entered in a hierarchical regression analysis, the variance in $\mathrm{ABS}$ was not significantly reduced by AoA, whereas MoA in the last step still explained a significant proportion of the variance.

The relationship between MoA, AoA, and ABS may be better conceived if we consider that the meaning of many abstract words is conveyed mainly through linguistic channels (see the bottom right panel of Figure 1), whereas information derived from perceptual cues is limited, which gives abstract concepts a less imageable "shape" as well. For example, the meaning of the concrete word apple is built mainly on perceptual groundings. Accordingly, the concept of an apple is more imageable and will be "picked up" earlier in life. However, the meaning of abstract words such as hope cannot be grasped through perceptual hints but needs to be mediated by linguistic and contextual information, which may not be promptly available at early stages of development. As a result, the concept of hope is perceived as less imageable and will be learned later, once all linguistic abilities are fully consolidated. 
In this deterministic framework, ABS turns out to be a concept-relative property, whereas MoA embraces both concept- and context-relative aspects of word meaning. AoA, instead, can be regarded as just an "effect" of MoA (Wauters, Tellings, \& van Bon, 2008). Thus, MoA appears to be more tightly bound to core elements of a concept, such as ABS, with respect to AoA, and can significantly "explain," to a certain degree, why the meaning of some words is judged as more or less abstract with respect to other ones.

We may thus conclude that MoA alone is able to quantify the degree of CNC or ABS of concepts, via the integration of information derived through experience and of that acquired through language, and may provide deeper insight into the dichotomy between the abstract and concrete domains. In particular, this dimension may be helpful in surmounting the fictitious "barricade" established by the physical characteristics of a concept. Finally, the correlations between our measures and those in the MRC database are high, suggesting that participants rely on the same type of information when rating $\mathrm{CNC}$, IMG, FAM, and AoA, even when norms are collected across languages. This is an important point supporting the reliability of the present norms.

\section{AUTHOR NOTE}

This research was supported by a European Union (FP6-2004-NESTPATH) Grant (028714) to S.F.C. Correspondence should be addressed to P. A. Della Rosa, San Raffaele Vita-Salute University, Via Olgettina, 20132, Milan, Italy (e-mail: dellarosa.pasquale@hsr.it).

\section{REFERENCES}

Altarriba, J., Bauer, L. M., \& Benvenuto, C. (1999). Concreteness, context availability, and imageability ratings and word associations for abstract, concrete, and emotion words. Behavior Research Methods, Instruments, \& Computers, 31, 578-602.

Balota, D. A., Yap, M. J., Cortese, M. J., Hutchison, K. A., KessLER, B., LOFTIS, B., ET AL. (2007). The English lexicon project. Behavior Research Methods, 39, 445-459.

Barca, L., Burani, C., \& Arduino, L. S. (2002). Word naming times and psycholinguistic norms for Italian nouns. Behavior Research Methods, Instruments, \& Computers, 34, 424-434.

Campos, A. (1990). Concreteness, imagery, emotionality, and interest values of words when meaning is controlled. Perceptual \& Motor Skills, 71, 603-610.

Coltheart, M. (1981). The MRC psycholinguistic database. Quarterly Journal of Experimental Psychology, 33A, 497-505.
Friendly, M., Franklin, P. E., Hoffman, D., \& Rubin, D. C. (1982). The Toronto Word Pool: Norms for imagery, concreteness, orthographic variables, and grammatical usage for 1,080 words. Behavior Research Methods \& Instrumentation, 14, 375-399.

Gilhooly, K. J., \& Logie, R. H. (1980). Age of acquisition, imagery, concreteness, familiarity and ambiguity measures for 1,944 words. Behavior Research Methods \& Instrumentation, 12, 395-427.

Gleitman, L. R., Cassidy, K., Nappa, R., Papafragou, A., \& Trueswell, J. C. (2005). Hard words. Language Learning \& Development, 1, 23-64.

Kousta, S., Della Rosa, P. A., Cappa, S. F., \& Vigliocco, G. (2007, August). The effect of mode of acquisition on lexical representation and processing. Paper presented at the 13th Annual Conference on Architectures and Mechanisms for Language Processing, Turku, Finland.

Nelson, D. L., \& Schreiber, T. A. (1992). Word concreteness and word structure as independent determinants of recall. Journal of Memory \& Language, 31, 237-260.

PaIvio, A. (1971). Imagery and verbal processes. New York: Holt, Rinehart \& Winston.

Paivio, A., Yuille, J. C., \& Madigan, S. A. (1968). Concreteness, imagery, and meaningfulness values for 925 nouns. Journal of Experimental Psychology, 76, 1-25.

Schwanenflugel, P. J., AKIN, C., \& LUh, W. (1992). Context availability and the recall of abstract and concrete words. Memory \& Cognition, 20, 96-104.

Schwanenflugel, P. J., Harnishfeger, K. K., \& Stowe, R. W. (1988). Context availability and lexical decisions for abstract and concrete words. Journal of Memory \& Language, 27, 499-520.

Setti, A., \& Caramelli, N. (2005). Different domains in abstract concepts. In B. Bara, L. Barsalou, \& M. Bucciarelli (Eds.), Proceedings of the XXVII Annual Conference of the Cognitive Science Society (pp. 1997-2002). Mahwah, NJ: Erlbaum.

Toglia, M. P., \& Battig, W. R. (1978). Handbook of semantic word norms. Hillsdale, NJ: Erlbaum.

Wauters, L. N., Tellings, A., \& van Bon, W. H. J. (2008). Mode of acquisition as a factor in deaf children's reading comprehension. Journal of Deaf Studies \& Deaf Education, 13, 175-192.

Wauters, L. N., Tellings, A., van Bon, W. H. J., \& van Haaften, W. A. (2003). Word of acquisition of word meanings: The viability of a theoretical construct. Applied Psycholinguistics, 24, 385-406.

Wiemer-Hastings, K., Krug, J., \& Xu, X. (2001). Imagery, context availability, contextual constraints and abstractness. In Proceedings of the 23rd Annual Meeting of the Cognitive Science Society (pp. 11061111). Hillsdale, NJ: Erlbaum.

\section{SUPPLEMENTAL MATERIALS}

The MoA norms and other psycholinguistic variables discussed in this article may be downloaded from http://brm.psychonomic-journals .org/content/supplemental, or from the "Abstract" Project Web site, www.abstract-project.eu.

(Manuscript received February 12, 2010; revision accepted for publication June 24, 2010.) 